\title{
Resistencia inicial a drogas antituberculosas en Buenaventura, Colombia
}

\begin{abstract}
César A. Moreira ${ }^{1}$, Héctor L. Hernández ${ }^{1}$, Nhora L. Arias ${ }^{1}$, Martha C. Castaño ${ }^{2}$, Beatriz E. Ferro ${ }^{1}$, Ernesto Jaramillo ${ }^{1}$

${ }^{1}$ Corporación Centro Internacional de Entrenamiento e Investigaciones Medicas, CIDEIM, Cali, Colombia. ${ }^{2}$ Secretaría de Salud del Valle, Cali, Colombia.

La tasa de notificación de tuberculosis pulmonar bacilífera en 2001 en Buenaventura, Colombia, fue de 66 por 100.000 habitantes. El pobre control de la tuberculosis en este municipio durante los últimos 10 años y el uso inadecuado de medicamentos de primera línea hace sospechar una elevada resistencia a estas drogas antituberculosas. En este artículo se presentan y discuten los resultados de dos encuestas de resistencia inicial a drogas antituberculosas de primera línea en personas con tuberculosis pulmonar realizadas en Buenaventura entre el $1^{\circ}$ de agosto de 1997 y el 31 de enero de 1998, y entre el 15 de noviembre de 2000 y el 15 de noviembre de 2001, con un intervalo de dos años y nueve meses. En las dos encuestas se utilizó el método de proporciones múltiples. Se logró aislar Mycobacterium tuberculosis en $93 \%$ y $55 \%$ de los casos nuevos de tuberculosis pulmonar diagnosticados en cada uno de los periodos estudiados. La resistencia inicial a cualquier medicamento antituberculoso fue de $25 \%(9 / 36)$ y $32 \%(23 / 72)$ en la primera y segunda encuesta, respectivamente. La multirresistencia inicial (definida como resistencia, al menos, a isoniacida y rifampicina) fue de $6 \%$ en ambas encuestas. Este porcentaje de resistencia encontrado demuestra la diseminación de cepas multirresistentes y destaca a la vigilancia epidemiológica de la resistencia a drogas antituberculosas como un componente esencial en el control de la enfermedad, por lo menos, en las zonas en las que el programa ha sido errático en el pasado. De esta manera, se podrán identificar las áreas en las que la estrategia DOTS deba ser reforzada con el uso de drogas de segunda línea (DOTS-plus).
\end{abstract}

Palabras claves: tuberculosis pulmonar, resistencia a drogas.

Initial drug resistance as a threat for tuberculosis control: the case of Buenaventura, Colombia

In 2001, Buenaventura, Colombia, the rate of smear-positive pulmonary tuberculosis was of 66 per 100.000 inhabitants. The poor control of the tuberculosis in this city during the last 10 years and the inadequate use of first-line anti-tuberculosis drugs was an opportune situation for the development of high resistance to these drugs. Two surveys of initial resistance to first-line antituberculosis drugs were conducted in new cases of pulmonary TB, in the city of Buenaventurathe first from August 1, of 1997 to January 31 of 1998 and the second from November 15, of 2000 to November 15, 2001. The method of multiple proportions was used to determine drug susceptibility. Mycobacterium tuberculosis was isolated in $93 \%$ and $55 \%$ of the new cases of lung TB during each respective period. The initial resistance to at least one drug was $25 \%$ (9/ $36)$ and $32 \%(23 / 72)$, respectively. The initial multi-drug resistance (defined as resistance to at least isoniazid and rifampicin) was $6 \%$ for both surveys. This demonstrates the dissemination of multidrug-resistant bacilli and shows the need for surveillance of resistance to antituberculosis drugs in control of the disease, particularly in those areas where the TB control program has been erratically applied. In areas where multi-drug resistant TB occurs, the control strategy should be enhanced with the careful introduction of second-line drugs.

Key words: lung tuberculosis, drug-resistant, multi-drug resistance. 
La multirresistencia a drogas antituberculosas (definida como la resistencia, al menos, a isoniazida y rifampicina) es ampliamente aceptada como una amenaza para el control de la tuberculosis en varios lugares del mundo $(1,2)$. Las drogas para el tratamiento de enfermos con tuberculosis multirresistente son menos eficaces, más costosas, más toxicas y requieren administración más prolongada que las drogas de primera línea (3-6). El número de drogas de segunda línea para tratar la tuberculosis multirresistente es limitado y las personas con esta enfermedad en los países menos desarrollados tienen un insuficiente acceso a ellas. La gran mayoría de estas personas fallecen a causa de la enfermedad, luego de haber diseminado bacilos multirresistentes en la comunidad. Los casos nuevos que han sido infectados por estos pacientes tendrán menores posibilidad de curación, lo cual hace más difícil el control de la epidemia. La pobre calidad de los programas de control de tuberculosis y las barreras que enfrenta el paciente para adherir al tratamiento, son factores que contribuyen a la creación y la transmisión de tuberculosis multirresistente $(2,7,8)$.

El Ministerio de Salud de Colombia notificó 20,1 casos de tuberculosis pulmonar bacilífera por 100.000 habitantes en el 2000 (9). A pesar de que dicha tasa es relativamente baja comparada con otros lugares del mundo (10), la notificación ha cambiado poco en los últimos 20 años debido a la insuficiente implementación de las actividades de control. A pesar de que la pobre adopción de las actividades de control predicen altos niveles de multirresistencia a la droga antituberculosa, los resultados de las encuestas de vigilancia epidemiológica realizadas no corresponden a lo esperado. La primera y la segunda encuestas nacionales de resistencia llevadas a cabo en 1999 y 2000 muestra una multirresistencia de 0,5\% (11) y $1,5 \%$, respectivamente (12).

Aunque los resultados de estas encuestas permiten afirmar que la resistencia a las drogas

Correspondencia:

César Moreira, Avenida 1N 3-03, Cali, Colombia.

cesarmoreira@ cideim.org.co

Recibido: 15/08/03; aceptado: 26/02/04 antituberculosas no es un problema para el control de la tuberculosis en Colombia, es necesario tener en cuenta que estos valores reportados corresponden a valores agregados de una muestra representativa para el país y, por tanto, no permiten identificar la situación de resistencia en un área específica.

Los reportes previos en el país hacen sospechar que existen regiones con una prevalencia e incidencia de resistencia a drogas superior al promedio nacional (13-15). Sin embargo, estos reportes no son representativos de la situación de resistencia a drogas en esas áreas. En el presente artículo se reportan y discuten los resultados de dos encuestas de resistencia a la droga antituberculosa de primera línea, realizadas en Buenaventura, Colombia. Este es un municipio con una notificación de tuberculosis superior a la tasa nacional y con un programa de control de tuberculosis ineficiente en los años previos a este estudio, por lo cual se estima que la resistencia a las drogas antituberculosas es superior a los valores promedio para el país.

\section{Materiales y metodos}

\section{Área de estudio}

Buenaventura es un puerto sobre la Costa Pacífica colombiana, puerta de entrada del $53 \%$ de las importaciones/exportaciones colombianas (16). Su población proyectada para el 2002 es de 271.401 habitantes (17). El 48\% de la población tiene sus necesidades básicas insatisfechas; el desempleo estimado es de $60 \%$ y el aseguramiento en salud es inferior al $40 \%$ (18).

La tasa de notificación y de mortalidad por tuberculosis pulmonar en 2001 es de 66 por 100.000 habitantes y 8,1 por 100.000 habitantes, respectivamente (Secretaría de Salud Pública Municipal de Buenaventura, comunicación personal). La estrategia DOTS para el control de la tuberculosis ha sido pobremente implementada en la década de los noventa. El porcentaje de abandono osciló entre $6 \%$ y $12 \%$ y el de curación no fue superior al $53 \%$; la detección de casos no sobrepasó el $24 \%$ en ese periodo. Las drogas de primera línea del tratamiento acortado supervisado (isoniacida, rifampicina, etambutol, estreptomicina 
y pirazinamida) han sido ampliamente usadas en este municipio desde 1980.

\section{Diseño del estudio}

Para determinar la prevalencia de la resistencia inicial a las drogas antituberculosas de primera línea, se analizaron los resultados de dos encuestas realizadas con una diferencia de 2 años y 9 meses. En ambas encuestas se solicitó al programa de control de tuberculosis de Buenaventura recolectar una muestra de esputo a todo mayor de 15 años con diagnóstico de tuberculosis pulmonar positiva en el examen directo, sin antecedentes de exposición previa por más de un mes a drogas antituberculosas de primera línea, previo al inicio del tratamiento acortado supervisado usado en Colombia $\left(2 \mathrm{HRZS} / 4 \mathrm{H}_{2} \mathrm{R}_{2}\right)$.

Se entrenó a los funcionarios de los puestos de salud del municipio en la obtención de dicha información y se revisaron los registros históricos del programa. Un integrante del grupo investigador entrevistó a cada uno de los pacientes para conocer este antecedente. Además, se les informó a los pacientes sobre los posibles resultados de la prueba diagnóstica, el tiempo para obtener un resultado y sus implicaciones terapéuticas y epidemiológicas.

La primera encuesta cubre un periodo de 6 meses ( $1^{\circ}$ de agosto de 1997 a 31 de enero de 1998) y la segunda un periodo de un año ( 15 de noviembre de 2000 al 15 de noviembre de 2001).

Las Secretarías de Salud Municipal de Buenaventura y de Salud del Valle ofrecieron la mejor terapia farmacológica disponible por el Programa Nacional de Control de la Tuberculosis, que incluyen drogas antituberculosas de segunda línea, a todos los pacientes diagnosticados con tuberculosis multirresistente en cada una de las encuestas reportadas en este estudio, de acuerdo con las recomendaciones internacionales existentes en ese momento.

\section{Cultivos y pruebas de sensibilidad}

La muestra de esputo obtenida de cada paciente fue enviada al Laboratorio de Micobacterias del Cideim en Cali, ubicado a $110 \mathrm{~km}$ de Buenaventura. Las muestras, depositadas en un frasco de tapa rosca y siguiendo las normas de bioseguridad, eran envueltas en papel absorbente, puestas en una bolsa plástica y empacadas en un recipiente hermético, el cual era embalado en contenedores especiales en condiciones de refrigeración, para que fueran procesadas en un tiempo no superior a 6 horas después de ser producida.

En el Cideim, las muestras fueron procesadas en el laboratorio de bioseguridad III; todas las muestras se descontaminaron con hidróxido de sodio/ N-acetil-L-cisteína y, posteriormente, se neutralizaron con solución tampón de fosfatos y se centrifugaron para su concentración. El sedimento se sembró por duplicado en dos clases diferentes de medio para el aislamiento de micobacterias: Lowenstein-Jensen y Middlebrook 7H11. Una vez obtenido crecimiento, se realizó la identificación de Mycobacterium tuberculosis mediante pruebas bioquímicas y se realizaron las pruebas de susceptibilidad por el método de las proporciones múltiples (19) con las siguientes drogas y concentraciones: isoniacida $(0,2 \mathrm{mg} / \mathrm{ml}$ y $1 \mathrm{mg} / \mathrm{ml})$, rifampicina $(1 \mathrm{mg} / \mathrm{ml})$, estreptomicina (2 $\mathrm{mg} / \mathrm{ml})$ y etambutol $(5 \mathrm{mg} / \mathrm{ml})$. Se determinó como resistencia la presencia de crecimiento igual o mayor al $1 \%$ en los cuadrantes con antibiótico, con respecto al control sin droga. Como controles se usaron las cepas H37Rv sensible a todos los medicamentos y la cepa AWC resistente a isoniacida y estreptomicina (20).

Las pruebas de susceptibilidad a drogas de primera línea realizadas en el laboratorio de CIDEIM tienen control de calidad certificado por el Laboratorio de Micobacterias del Instituto de Salud Pública de Chile, laboratorio supranacional de referencia para Colombia. La correlación obtenida para la rifampicina y la isoniacida fue de $100 \%$.

\section{Resultados}

El cuadro 1 muestra el origen de los aislamientos de $M$. tuberculosis en los que fue posible practicar prueba de susceptibilidad. Para la primera encuesta, se diagnosticaron 46 casos nuevos de tuberculosis pulmonar bacilífera; en $43(93 \%)$ de ellos, se aisló $M$. tuberculosis y en 36 (78\%) fue 
Cuadro 1. Selección de los pacientes con tuberculosis pulmonar bacilífera en el examen directo con prueba de susceptibilidad a los medicamentos antituberculosos en cada una de las dos encuestas de resistencia realizadas en Buenaventura (1997-1998 y 2000-2001).

\begin{tabular}{|c|c|c|}
\hline & $\begin{array}{c}\text { Primera } \\
\text { encuesta } \\
(n=46)\end{array}$ & $\begin{array}{c}\text { Segunda } \\
\text { encuesta } \\
(n=134)\end{array}$ \\
\hline Muestras de esputo obtenidas & $46(100 \%)$ & $84(63 \%)$ \\
\hline Aislamientos de $M$. tuberculosis & $43(93 \%)$ & $74(55 \%)$ \\
\hline $\begin{array}{l}\text { Casos con prueba de } \\
\text { susceptibilidad }\end{array}$ & $36(78 \%)$ & $72(54 \%)$ \\
\hline
\end{tabular}

posible realizar prueba de susceptibilidad. Para la segunda encuesta, se diagnosticaron 134 casos nuevos de tuberculosis pulmonar bacilífera; se enviaron para cultivo 84 (63\%); se aisló $M$. tuberculosis en 74 (55\%) y se realizó prueba de susceptibilidad en $72(54 \%)$. A 50 (37\%) pacientes en la segunda encuesta no se les pudo evaluar debido a que ingresaron al programa tras el inicio del tratamiento en otras instituciones, no suministraron muestra adecuada, el crecimiento fue insuficiente o hubo contaminación.

El cuadro 2 muestra los resultados de las pruebas de susceptibilidad a las drogas antituberculosas de primera línea obtenida en los dos grupos de pacientes estudiados. La resistencia inicial a cualquier droga fue de $25 \%$ (9/36) y de $32 \%$ (23/ 72) en la primera y segunda encuesta, respectivamente. La multirresistencia inicial fue de $6 \%$ en ambas encuestas. No se encontraron diferencias significantes en los valores de resistencia determinados para cada una de las drogas, excepto para estreptomicina $(p=0,0143)$.

\section{Discusión}

La multirresistencia a las drogas antituberculosas es ampliamente aceptada como una seria amenaza para el control de la enfermedad, especialmente en lugares donde es particularmente elevada como Estonia (14,1\%), la provincia de Henan en China $(10,8 \%)$, Letonia $(9 \%)$, las provincias rusas Ivanovo (9\%) y Tomsk $(6,4 \%)$ e Irán (5\%) (11). Los resultados de las dos encuestas de resistencia a las drogas antituberculosas realizadas entre 1997 y 2001 en Buenaventura y presentados en este artículo muestran una multirresistencia inicial de $6 \%$.

La validez de estos resultados está basada en la consistencia del método usado para recolectar las muestras y en la calidad certificada del laboratorio que realizó las pruebas de susceptibilidad. Sin embargo, algunos factores afectan la validez de los hallazgos. En la segunda encuesta se obtuvo prueba de susceptibilidad en una proporción menor de casos nuevos respecto a la primera encuesta debido a que operó un sesgo de selección: no se obtuvo aislamiento en $37 \%$ de los pacientes ya que éstos habían iniciado tratamiento en otra unidad de salud. Sin embargo, no hay razones para considerar que dichos pacientes correspondan a una población diferente de aquella en la que sí se obtuvo muestra y, por tanto, no se considera que este hecho afecte de manera significativa la representatividad de la muestra final obtenida y la validez de los resultados.

En Buenaventura, como en muchos otros lugares, existe estigma asociado con la tuberculosis. Este estigma puede influir en la admisión por parte de

Cuadro 2. Distribución de la resistencia inicial a medicamentos antituberculosos en Buenaventura, en pacientes con tuberculosis pulmonar bacilífera en los periodos de 1 de agosto de 1997 a 31de enero de 1998 (Grupo 1), y del 15 de noviembre de 2000 al 15 de noviembre de 2001 (Grupo 2).

\begin{tabular}{|c|c|c|c|c|c|}
\hline Resistencia a droga & $\begin{array}{l}\text { Grupo } 1 \\
(n=36)\end{array}$ & $\begin{array}{c}\text { Grupo } 2 \\
(n=72)\end{array}$ & $\chi^{2}$ & IC & $p$ \\
\hline A uno o más medicamentos & $9(25 \%)$ & $23(32 \%)$ & 1,05 & $-0,276-0,082$ & 0,153 \\
\hline Rifampicina & $3(8 \%)$ & $4(6 \%)$ & 0,31 & $-0,077-0,132$ & 0,290 \\
\hline Isoniacida & $8(22 \%)$ & $16(22 \%)$ & 0,00 & $-0,151-0,179$ & 0,500 \\
\hline Estreptomicina & $2(6 \%)$ & $16(22 \%)$ & 4,80 & $-0,288--0,045$ & 0,014 \\
\hline Etambutol & $0(0 \%)$ & $1(1 \%)$ & 0,50 & $-0,041-0,013$ & 0,239 \\
\hline Multirresistencia & $2(6 \%)$ & $4(6 \%)$ & 0,00 & $-0,092-0,092$ & 0,500 \\
\hline
\end{tabular}


los pacientes de haber tenido tuberculosis en el pasado o haber estado recibiendo drogas antituberculosas. Aunque los funcionarios del programa de control de tuberculosis fueron entrenados para preguntar a los pacientes la exposición previa a droga en una enfermedad, es posible que algunos pacientes hayan negado la exposición previa a drogas por el estigma asociado a la enfermedad en este municipio (Arias NL, et al., manuscrito en preparación) y, por tanto, el resultado de la resistencia inicial a las drogas aquí presentado sea superior a la realidad. Sin embargo, se estima que este factor que afecta cualquier encuesta de resistencia a drogas antituberculosas, operó de la misma manera en ambas encuestas, lo cual permite comparar los resultados de las mismas.

El breve intervalo entre ambas encuestas y la ausencia de una intervención para el control de la tuberculosis multirresistente explica el que no haya diferencia significante en la prevalencia de resistencia inicial para las drogas evaluadas. La estreptomicina es una excepción; sin embargo esta diferencia es estadísticamente significante aunque tiene una validez limitada pues la prueba de susceptibilidad por el método de proporciones múltiples tiene un valor predictivo positivo bajo para esta droga.

El valor encontrado de multirresistencia inicial en Buenaventura en este estudio tiene significado en el contexto de los hallazgos de las investigaciones hechas en este municipio y reportados en otras publicaciones $(15,21)$. En estos reportes se describen errores en el manejo de las drogas antituberculosas por parte de los médicos e inadecuada implementación de la estrategia DOTS como factores causantes del desarrollo de multirresistencia en este municipio. Uno de estos estudios demostró, mediante la aplicación de técnicas moleculares, la transmisión de cepas multirresistentes en el área urbana del municipio (15). Los hallazgos del presente estudio justifican la realización de investigaciones que permitan discernir los patrones de circulación de cepas multirresistentes para poder ajustar las actividades de control al contexto de la epidemiología local de la tuberculosis.
Un componente fundamental de la estrategia DOTS para el control de la tuberculosis es el tratamiento supervisado con rifampicina, isoniacida, pirazinamida, en asocio con estreptomicina o etambutol. Este tratamiento puede ser inadecuado en áreas con elevada resistencia a estas drogas, pues las tasas de curación son inferiores y la morbilidad y mortalidad asociada es mayor (22-26).

Las repercusiones de un esquema inadecuado no sólo puede afectar de manera negativa el resultado final del tratamiento sino también la situación de resistencia ya existente. En efecto, la evidencia sugiere que la administración de esquemas de tratamiento acortados en pacientes que tienen predominio de cepas resistentes resulta en la amplificación de la resistencia a drogas ya existente (23-25). En el caso de Buenaventura, esta situación es plausible en la medida que, de acuerdo con la resistencia inicial a la isoniacida encontrada en este estudio, $22 \%$ de los pacientes nuevos con tuberculosis pulmonar estarían recibiendo monoterapia con rifampicina durante la fase de continuación del tratamiento acortado supervisado, la manera más eficaz para desarrollar resistencia a cualquier droga antituberculosa.

Los hallazgos de este estudio confirman la necesidad de realizar estudios de resistencia en lugares donde hay antecedentes de una inadecuada implementación de la estrategia DOTS. Sólo mediante la información obtenida por estos estudios es posible evaluar la conveniencia de incorporar a la estrategia DOTS, medidas para realizar diagnóstico temprano de casos multirresistentes e incluir drogas de segunda línea en los esquemas de tratamiento (DOTS-plus) (27). El diagnóstico temprano de la multirresistencia y su tratamiento con drogas de segunda línea dentro de la estrategia DOTS es una actividad compleja y más costosa que el manejo de la tuberculosis en pacientes sensibles a las drogas de primera línea; sin embargo, puede ser la manera más efectiva para controlar la tuberculosis multirresistente $(28,29)$.

\section{Agradecimientos}

Los autores agradecen la colaboración proporcionada por Nancy S. Henríquez y los 
funcionarios de la Secretaría de Salud Pública Municipal de Buenaventura en la recolección de las muestras; a los miembros del Laboratorio de Micobacterias del Cideim durante la realización del estudio; y a M. Hazbón, K. Laserson, N. Saravia y dos revisores anónimos por sus valiosos comentarios sobre versiones previas de esta manuscrito. Este trabajo fue financiado por el Ministerio de Salud de Colombia y la Secretaría de Salud del Valle del Cauca.

\section{Referencias}

1. World Health Organization. The WHO/IUATLD global project on anti-tuberculosis drug resistance surveillance. Anti-tuberculosis drugs resistance in the world. Report No.2 Prevalence and trends. Geneva: World Health Organization; 2000.

2. Pablos-Méndez A, Raviglione MC, Laszlo A, Binkin N, Rieder HL, Bustreo F., et al. Global surveillance for antituberculosis-drug resistance. N Engl J Med 1998;338:1641-9

3. Goble M, Iseman MD, Madsen LA, Waite D, Ackerson L, Horsburgh CR Jr, et al. Treatment of 171 patients with pulmonary tuberculosis resistant to isoniazid and rifampin. N Eng J Med 1993;328:527-32.

4. CDC. Initial therapy for tuberculosis in the era of multidrug resistance. Morb Mortal Week Rep 1993;42/ No.RR-7.

5. Crofton J, Chaulet P, Maher D. Directrices para el tratamiento de la tuberculosis farmacorresistente. Ginebra: Organización Mundial de la Salud; 1997.

6. PIH. A DOTS-plus handbook: guide to the communitybased treatment for MDR TB. Boston: Partners in Health; 2002.

7. Farmer P, Kim JY. Community based approaches to the control of multidrug resistant tuberculosis: introducing "DOTS-plus". BMJ 1998;317:671-4.

8. Jaramillo E. Encompassing prevention with treatment: the path for a long-lasting control of tuberculosis. Social Science and Medicine 1999;49:393-404.

9. Ministerio de Salud, Instituto Nacional de Salud. Tuberculosis Boletín Epidemiológico Semanal Noviembre 10-17, 2001.

10. World Health Organization. WHO report 2003. Global tuberculosis control. Surveillance, planning, financing. Geneva: World Health Organization; 2003.

11. Espinal M, Laszlo A, Simonsen L, Boulabahl F, Kim $\mathbf{S}$, Reniero A, et al. Global trends in resistance to antituberculosis drugs. N Engl J Med 2001;344:1294303.

12. León CI, Sierra CR, Naranjo ON, Garzón MC, Guerrero MI. Segundo estudio nacional de resistencia primaria del Mycobacterium tuberculosis a las drogas en Colombia. Resúmenes, Sexto Congreso Colombiano de Enfermedades Infecciosas. Infectio 2002;6:A-12.

13. Crespo MP, Vélez JD. Aislamiento de M. tuberculosis (Mtb) y perfil de resistencia en un hospital de tercer nivel en Cali, Colombia. Infectio 1999;3:19.

14. Bermúdez A, Mattar A, Visbal J. Descripción de la susceptibilidad antimicrobiana de cepas de M. tuberculosis aisladas en Bogotá D.C. Resúmenes, Cuarto Congreso Colombiano de Enfermedades Infecciosas. Infectio 1999;3:36.

15. Laserson KF, Osorio L, Sheppard JD, Hernández $\mathrm{H}$, Benítez AM, Brim S, et al. Clinical and programmatic mismanagement rather than community outbreak as the cause of chronic, drug-resistant tuberculosis in Buenaventura, Colombia, 1998. Int J Tuberc Lung Dis 2000;4:673-83.

16. Sociedad Portuaria de Buenaventura. Reporte anual. Buenaventura: Sociedad Portuaria de Buenaventura Ltda.; 1999.

17. Departamento Nacional de Estadística. Colombia, 1993. Bogotá: Departamento Nacional de Estadística; 1993.

18. Secretaría de Salud Pública Municipal de Buenaventura. Plan Local de Salud 2000. Buenaventura: Secretaría de Salud Pública Municipal de Buenaventura; 2000.

19. Kent PT, Kubica G. Public health mycobacteriology: a guide for the level III laboratory. Atlanta: Centers for Disease Control; 1985. p.166-74.

20. Wanger A, Mills K. Testing of Mycobacterium tuberculosis susceptibility to ethambutol, isoniazid, rifampin, and streptomycin by using Etest. J Clin Microbiol 1996;34:1672-6.

21. CDC. Acquired multidrug-resistant tuberculosis Buenaventura, Colombia, 1998. Morb Mortal Week Rep 1998;47:759-61.

22. Espinal M, Kim SJ, Suarez PG, Kam KM, Khomenko AG, et al. Standard short-course chemotherapy for drug resistant tuberculosis: treatment outcomes in six countries. JAMA 2000;283:2537-45.

23. Farmer P, Bayona J, Becerra M, Furin J, Shin S. Effect of administering short-course, standarizaded regimens in individuals infected with drug-resistant Mycobacterium tuberculosis strains. Eur J Clin Microbiol Infect Dis 2000;19:132-6.

24. Quy HTW, Lan NTN, Borgdorff M, et al. Drug resistance among failure and relapse cases of tuberculosis: is the standard retreatment regimen adequate? Int $\mathrm{J}$ Tuberc Lung Dis 2003;7:631-6.

25. Coninx R, Mathieu C, Debacker M, Mirzoev F, Ismaelov A, de Haller R, et al. First-line tuberculosis therapy and drug-resistant Mycobacterium tuberculosis in prisons. Lancet 1999;353:969-73. 
26. García-García ML, Ponce de León A, JiménezCorona ME, Jiménez-Corona A, Palacios-Martínez $\mathrm{M}$, Balandrano-Campos $\mathrm{S}$, et al. Clinical consequences and transmissibility of drug-resistant tuberculosis in southern Mexico. Arch Intern Med 2000;160:630-6.

27. World Health Organization. Scientific panel of the working group on DOTS-plus for MDR-TB. Guidelines for establishing DOTS-plus pilot projects for the management of multidrug-resistant tuberculosis (MDR-TB).
Geneva: World Health Organization; 2000. (WHO/CDS/ TB/2000.279).

28. Tahaoglu K, Torun T, Sevim T, Atac G, Kir A, Karasulu $\mathrm{L}$, et al. The treatment of multidrug-resistant tuberculosis in Turkey. N Engl J Med 2001;345:170-4.

29. Farmer P, Kim YJ, Mitnick C, Bayona J, Shin S, Furin $\mathrm{J}$, et al. Community-based therapy for multidrugresistant tuberculosis in Lima, Peru. $\mathrm{N}$ Engl $\mathrm{J}$ Med 2003;348:119-28. 